\title{
Holonomy, the Quantum Adiabatic Theorem, and Berry's Phase
}

\author{
Barry Simon \\ Departments of Mathematics and Physics, California Institute of Technology, Pasadena, California 91125
}

(Received 18 October 1983)

\begin{abstract}
It is shown that the "geometrical phase factor" recently found by Berry in his study of the quantum adiabatic theorem is precisely the holonomy in a Hermitian line bundle since the adiabatic theorem naturally defines a connection in such a bundle. This not only takes the mystery out of Berry's phase factor and provides calculational simple formulas, but makes a connection between Berry's work and that of Thouless et al. This connection allows the author to use Berry's ideas to interpret the integers of Thouless et al. in terms of eigenvalue degeneracies.
\end{abstract}

PACS numbers: $03.65 . \mathrm{Db}, 02.40 .+\mathrm{m}$

Vector bundles and their integral invariants (Chern numbers) are already familiar to theoretical physicists because of their occurrence in classical Yang-Mills theories. Here I want to explain how they also enter naturally into nonrelativistic quantum mechanics, especially in problems connected with condensed matter physics. If one has a Hermitian operator $\tilde{H}(x)$ depending smoothly on a parameter $x$, with an isolated nondegenerate eigenvalue $\boldsymbol{E}(x)$ depending continuously on $x$, then $\{(x, \varphi) \mid \tilde{H}(x) \varphi=E(x) \varphi\}$ defines a line bundle over the parameter space. I will show that the twisting of this line bundle affects the phase of quantum mechanical wave functions.

Berry, in a beautiful recent paper, ${ }^{1}$ discovered a striking phenomenon in the quantum adiabatic theorem..$^{2}$ That theorem says ${ }^{3}$ that if $H(t), 0 \leqslant t$ $\leqslant 1$, is a family of Hermitian Hamiltonians, depending smoothly on $t$, and if $E(t)$ is a smooth function of $t$ which is a simple eigenvalue of $H(t)$ isolated from the rest of the spectrum of $H(t)$ for each $t$, then the solution $\psi_{T}(s)$ of the time-dependent Schrödinger equation

$$
i d \psi_{T}(s) / d s=H(s / T) \psi_{T}(s)
$$

with $\psi_{T}(0)=\varphi_{0}$ where $H(0) \varphi_{0}=E(0) \varphi_{0}$ has the property that as $T-\infty, \psi_{T}(T)$ approaches the eigenvector $\varphi_{1}$ of $H(1)$ with $H(1) \varphi_{1}=E(1) \varphi_{1}$. Berry asked the following question: Suppose that $\tilde{H}(x)$ is a multiple-parameter family and that $C(t)$ is a closed curve in parameter space, so that $\tilde{H}(C(t)) \equiv H(t)$ obeys the hypotheses of the adiabatic theorem. Then that theorem says that $\varphi_{1}$ is just a phase factor times $\varphi_{0}$ and Berry asks, "What phase factor?" Surprisingly, the "obvious" guess

$$
\varphi_{1}=\exp \left[-i \int_{0}^{T} E(s / T) d s\right] \varphi_{0}
$$

is wrong; rather, Berry finds

$$
\varphi_{1}=\exp \left[-i \int_{0}^{T} E(s / T) d s\right] \exp [i \gamma(C)] \varphi_{0},
$$

where $\gamma(C)$ is an extra phase which Berry extensively studies, and which he suggests could be experimentally measured. ${ }^{4}$

The purpose here is first to advertise what Berry calls a "remarkable and rather mysterious result," but more basically to try to take the mystery out of it by realizing that $\gamma$ is an integral of a curvature so that Berry's phenomenon is essentially that of holonomy which is becoming quite familiar to theoretical physicists. ${ }^{5}$ This realization will allow us a more compact formula than that used principally by Berry, and one that is easier to compute with. Most importantly, it will give a close mathematical relationship between his work and that of Thouless etal., ${ }^{6}$ so that Berry's interesting analysis of the relation of degeneracy to $\gamma(\boldsymbol{E})$ will allow us to interpret the $\mathrm{TKN}^{2}$ integers in a new and interesting way.

To explain that $\gamma$ is a holonomy, I begin by replacing $H(s)$ by $H(s)-E(s)$ which produces a trivial, computable phase change in the solution $\psi_{T}(s)$ of (1). Thus, without loss, we can take $E(s)$ $\equiv 0$. Define $\eta_{T}(s)=\psi_{T}(s T)$ so that $\eta$ solves

$$
i d \eta_{T}(s) / d s=T H(s) \eta_{T}(s),
$$

and the adiabatic theorem says that $\eta_{T}(s)$ has a limit $\eta(s)$ with

$$
H(s) \eta(s)=0
$$

[since we have taken $E(s)=0$ ]. If now $\tilde{H}(x)$ is a multiparameter family of Hermitian Hamiltonians, so that in some region $\boldsymbol{M}$ of parameter space, 0 is an isolated nondegenerate eigenvalue, then given any curve $C(t)$ and any choice $\eta_{0}$ of normalized zero-energy eigenvector of $H(C(0))$ (i.e., a choice of phase), the adiabatic limit yields a way of transporting $\eta_{0}$ along the curve $C(t)$, i.e., a connection. In this way, (2) is just an expression of the holonomy associated to this connection. So far this is just fancy words to de- 
scribe Berry's discovery. However, there is a mathematically natural connection already long known in the situation of distinguished lines in Hilbert space. For given $x$, let $X_{x}$ denote the zero-energy eigenspace for $\tilde{H}(x)$. This yields a line bundle over the parameter space which, since it is embedded in $M \times \mathcal{H}$, has a natural Hermitian connection, studied, e.g., by Bott and Chern. ${ }^{7}$ In this connection, one transports a vector $\beta_{0}$ along a curve $C(t)$ so that $\beta(t)$ obeys $\langle\beta(t+\delta t), \beta(t)\rangle=1+O\left((\delta t)^{2}\right)$. I claim that $\eta(s)$ precisely obeys this condition; formally, one can argue that

$$
\begin{aligned}
\left(\eta(s), \frac{d \eta(s)}{d s}\right) & =\lim _{T \rightarrow \infty}\left(\eta(s), \frac{d \eta_{T}(s)}{d s}\right) \\
& =\lim _{T \rightarrow \infty}\left(\eta(s),-i T H(s) \eta_{T}(s)\right)=0
\end{aligned}
$$

by (4). One can give a rigorous proof just using the convergence of $\eta$ without worrying about the question of convergence of derivatives. ${ }^{8}$ Thus the connection given by the adiabatic theorem [when $E(s) \equiv 0$ ] is precisely the conventional one for embedded Hermitian bundles and $\gamma$ is the conventional integral of the curvature which is just the Chern class of the connection. In particular, $\gamma$ only depends on the $X_{x}$ 's, not other aspects of $\tilde{H}(x)$. This means that one has a simple compact formula ${ }^{9}$ for $\gamma$ :

$$
\gamma(C)=\int_{s} V,
$$

where $S$ is any oriented surface in $M$ with $\partial S=C$ and $V$ can be defined in terms of an arbitrary smooth choice, ${ }^{10} \varphi(x)$, of unit vectors in $X_{x}$ by

$$
V=i(d \varphi, d \varphi) \text {, }
$$

which is shorthand for the two-form ${ }^{11}$

$$
V=\sum_{i<j} \operatorname{Im}\left(\partial \varphi / \partial x_{i}, \partial \varphi / \partial x_{j}\right) d x_{i} \wedge d x_{j}
$$

written in terms of local coordinates. The formula that Berry used has the advantage over (5) of being manifestly invariant under phase changes of $\varphi(x),,^{12}$ but it appears to depend on details of $\tilde{H}(x)$ and not just on the spaces $X_{x}$. Moreover, since it has a sum over intermediate states, it could be difficult to compute in general, although in his examples it is easy to compute, since the sum over intermediate state in these examples is finite. Even in these examples, (5) can be very easy to use in computations. ${ }^{13}$

Equation (5) shows that $V=0$ if one can choose the $\varphi(x)$ to be all simultaneously real. Thus, the phenomena we discuss here are only present in magnetic fields or some other condition producing a nonreal Hamiltonian.

As an example of significance below which is also considered by Berry, let $M=R^{3} \backslash\{0\}$ and given $x \in M$, let $H(x)=\overrightarrow{\mathrm{x}} \cdot \overrightarrow{\mathrm{L}}$ where $\mathrm{L}$ is a spin-S spin on $C^{2 S+1}$. Then all eigenvalues are nondegenerate and we can, for each $m=-S,-S+1, \ldots, S$, compute a $V_{m}(\vec{x})$ associated to the eigenvalue $|\vec{x}| m$. By rotation covariance, $V_{m}(\vec{x})$ must be a function of $|\vec{x}|$ times the area form $A(\vec{x})$ on the sphere of radius $\overrightarrow{\mathrm{x}}$. Thus, we need only compute $V_{m}$ at $\overrightarrow{\mathrm{x}}=(0,0, z)$. If $|m\rangle$ is the vector with $L_{z}|m\rangle$ $=m|m\rangle$, then for $\overrightarrow{\mathrm{x}}$ near $(0,0, z)$, we can take

$$
\varphi(\vec{x})=\exp \left[i\left(\frac{x}{z} L_{y}-\frac{y}{z} L_{x}\right)+O\left(x^{2}+y^{2}\right)\right]|m\rangle
$$

so that

$$
\begin{aligned}
& d \varphi=i z^{-1}\left[d x L_{y}-d y L_{x}\right]|m\rangle, \\
& \begin{aligned}
(d \varphi, d \varphi) & =z^{-2} d x \wedge d y\left\langle m\left|\left[L_{y}, L_{x}\right]\right| m\right\rangle \\
& =i z^{-2} m d x \wedge d y,
\end{aligned}
\end{aligned}
$$

and thus, returning to general $x$,

$$
V_{m}(\overrightarrow{\mathrm{x}})=m|\overrightarrow{\mathrm{x}}|^{-2} A(\overrightarrow{\mathrm{x}}) \text {. }
$$

In particular, if $S$ is any sphere $\mathrm{s}^{14}$ about the origin,

$$
(2 \pi)^{-1} \int_{S} V_{m}(\vec{x})=2 m
$$

is an integer. This is no coincidence: If $C$ is a clockwise circuit around the equator of the sphere $S$, which breaks up $S$ into two hemispheres $S_{+}, S_{-}$ with $\partial S_{ \pm}= \pm C$, then

$$
\exp [i \gamma(C)]=\exp \left(i \int_{S_{+}} V\right)=\exp \left(-i \int_{S_{-}} V\right)
$$

so that $\int_{S} V$ must be $2 \pi$ times an integer. We therefore see the familiar quantization of the integral of the Chern class, $V$, of the bundle, as a consistency condition on the holonomy, a standard fact.

Thouless etal., in their deep analysis of the quantized Hall effect, ${ }^{6}$ considered a band of a two-dimensional solid in magnetic field, so that for each $k$ in $T^{2}$, the Brillouin zone, the corresponding band energy is nondegenerate. If $\varphi(k)$ is the corresponding eigenvector, then $(i / 2 \pi)$ $\times \int_{T^{2}}(d \varphi, d \varphi)$ is an integer, the $\mathrm{TKN}^{2}$ integer of the band. Using the source ${ }^{15}$ analogy of Berry, ${ }^{1}$ we can "interpret" these integers. Suppose the band under consideration is the $n$ th; and suppose an arbitrary smooth interpolation, $\tilde{H}(k),{ }^{16}$ of the band Hamiltonian $H(k)$ is given from the surface of the torus into the solid torus $\tilde{T}$, i.e., $\tilde{H}(k)$ is defined for $\tilde{k}$ in $\tilde{T}$ and equals $H(k)$ on the surface. 
The Wigner-von Neumann theorem ${ }^{17}$ says that generically, ${ }^{16}$ the $n$th band is only degenerate for isolated points $\left\{p_{i}\right\}_{i=1}^{l}$ in $\tilde{T}$. One can define $V$ on $\tilde{T}$ with these points removed, and since $d V=0$, Gauss' theorem assures us that the integral of $V$ over the torus is just the same as its integral over little spheres about the degeneracies. Each sphere has a "charge" associated with it which is $\frac{1}{2} q_{i}$ with $q_{i}$ an integer, and $\sum q_{i}$ is the $\mathrm{TKN}^{2}$ integer.

It is worthwhile to expand slightly on this picture..$^{18}$ Consider a matrix family $M(\overrightarrow{\mathrm{x}})$, depending smoothly on these parameters. If all eigenvalues of $M\left(\overrightarrow{\mathrm{x}}_{0}\right)$ are nondegenerate, we say that $\overrightarrow{\mathrm{x}}_{0}$ is a regular point. $\overrightarrow{\mathrm{x}}_{0}$ is a normal singular point if and only if (i) only one eigenvalue is degenerate and its multiplicity is 2; (ii) the degeneracy is removed to first order for any line through $\overrightarrow{\mathrm{x}}_{0}$. If 0 is a normal singular point and $P$ is the projection onto the degenerate eigenvalues of $\boldsymbol{M}(0)$, then for $\overrightarrow{\mathrm{x}}$ near zero,

$$
P M(\overrightarrow{\mathrm{x}}) P-P M(0) P=\overrightarrow{\mathrm{a}} \cdot \overrightarrow{\mathrm{x}} P+\overrightarrow{\mathrm{B}} \cdot \overrightarrow{\mathrm{x}}+O\left(x^{2}\right)
$$

where $\overrightarrow{\mathrm{B}}$ is a vector of traceless operators on $P$. Picking a basis for the range of $P_{,}^{19}$ we can write $\overrightarrow{\mathrm{B}} \cdot \overrightarrow{\mathrm{X}}=\vec{\sigma} \cdot \boldsymbol{C} \overrightarrow{\mathrm{X}}$ where $C$ is a $3 \times 3$ matrix and $\vec{\sigma}$ are the usual Pauli matrices in the basis. The condition on removal of degeneracy says that $\operatorname{det}(C)$ $\neq 0$. The Hermiticity of $M$ implies $\operatorname{det}(C)$ is real. I call the sign of $\operatorname{det}(C)$ the signature $\sigma\left(\overrightarrow{\mathrm{x}}_{0}\right)$ of the normal singular point. With use of a deformation argument and the example discussed above (with spin $S=\frac{1}{2}$ ), it is not hard to show that if the $n$th and $(n+1)$ st levels are degenerate at $x_{0}$, and if $V_{j}$ is the Chern class associated to the $j$ th level, then for a small sphere $S$ about $x_{0}$, we have that

$$
(2 \pi)^{-1} \int_{S} V_{n+1}=\sigma\left(x_{0}\right) ;(2 \pi)^{-1} \int_{S} V_{n}=-\sigma\left(x_{0}\right) .
$$

If $M$ is a smooth, regular matrix family on $T^{2}$ and $\tilde{M}$ is a smooth interpolation to $\tilde{T}$ with only normal singular points, ${ }^{16}$ then the $n$th $\mathrm{TKN}^{2}$ integer is exactly equal to a weighted sum of singular points: Points where the $n$th level is nondegenerate get weight zero, those where it is degenerate with the next lower level get weight $\sigma(p)$ where $\sigma$ is the signature of $p$, and those where it is degenerate with the next higher level get weight $-\sigma(p) .^{20}$

I conclude with a mathematical remark: I have shown how the Chern integers associated to certain line bundles can be understood in terms of singularities of interpolations of the bundle. It would be interesting to extend this picture to gen- eral vector bundles.

It is a pleasure to thank D. Robinson and N. Trudinger for the hospitality of the Australian National University, where this work was done, M. Berry for telling me of his work, and B. Souillard for the remark (via $M$. Berry) that there must be a connection between Berry's work and that of $\mathrm{TKN}^{2}$. This research was partially supported by the National Science Foundation through Grant No. MCS-81-20833.

\footnotetext{
${ }^{1}$ M. V. Berry, to be published; see also Proceedings of the Como Conference on Chaos, 1983 (to be published).

${ }^{2}$ See T. Kato, J. Phys. Soc. Jpn. 5 , 435-439 (1950);
} A. Messiah, Quantum Mechanics (North-Holland, Amsterdam, 1962), Vol. 2.

${ }^{3}$ There are, in the infinite-dimensional case, also domain conditions if $H(t)$ is unbounded. We ignore these in our discussion. For purposes of this note, one can think of finite-dimensional cases.

${ }^{4}$ Since $\int_{0}^{T} E(s / T) d s=T \int_{0}^{1} E(s) d s$ will be very large in the limit $T \rightarrow \infty$ unless $\int_{0}^{1} E(s) d s=0$, it may be difficult to set up the experiment in such a way that the "dynamic phase" $\int{ }_{0}^{T} E(s / T) d s$ does not wash out $\gamma(C)$.

${ }^{5}$ See T. Eguchi et al., Phys. Rep. 66, 213 (1980); Y. Choquet-Bruhat et al., Analysis Manifolds and Physics (North-Holland, Amsterdam, 1983).

${ }^{6} \mathrm{D}$. Thouless, M. Kohmoto, M. Nightingale, and M. den Nijs, Phys. Rev. Lett. 49 , 405 (1982), designated as $\mathrm{TKN}^{2}$.

${ }^{7}$ R. Bott and S. Chern, Acta Math. 114, 71 (1965).

${ }^{8}$ Pick $f$ a smooth function of compact support and compute

$$
\int f(s)\left(\eta(s), \frac{d \eta}{d s}\right) d s=\lim _{T \rightarrow \infty} \int f(s)\left(\eta_{T}(s), \frac{d \eta}{d s}\right) .
$$

Integrate by parts and get one term $\left(d \eta_{T} / d s, \eta\right)$ which is zero by (3) and (4) and one term

$$
\lim _{T \rightarrow \infty} \int f^{\prime}(s)\left(\eta_{T}, \eta\right)=\int f^{\prime}(s)(\eta, \eta) d s=\int f^{\prime}(s) d s=0 .
$$

${ }^{9}$ While one gets (5) by appealing to the abstract theory, it is quite easy to compute. If $\eta(t)=e^{i \theta(t)} \varphi(t)$, then $(\eta(s), d \eta / d s)=0$ becomes $(\varphi(t), d \varphi / d t)+i \dot{\theta}=0$, so that $\gamma(C)=\oint_{C} i(\varphi, d \varphi)=\int_{S} i(d \varphi, d \varphi)$ by Stokes' theorem.

${ }^{10}$ If $M$ has "holes," it may not be possible to choose $\varphi$ globally, but since (5) is phase invariant, one need only make a choice in a neighborhood of any given point. If $S$ is the image of a disk (as it typically is), one can always make a global choice.

${ }^{11}$ This formula appears as Eq. (7b) in Ref. 1 but only in passing; surprisingly, it is not used again. For example, with it, the one-page calculation in Ref. 1 that $d V=0$ is trivial from $d^{2}=0$.

${ }^{12}$ It is an easy calculation that (5) is invariant under $\varphi(x) \rightarrow e^{i \theta(x)} \varphi(x)$ since the normalization condition 
$(\varphi, \varphi)=1$ implies that $(\varphi, d \varphi)+(d \varphi, \varphi)=0$. Avron, Seiler, and Simon [J. Avron, R. Seiler, and B. Simon, to be published; see also J. Avron et al., Phys. Rev. Lett. 51, 51-53 (1983)] give a simple manifestly phaseinvariant form for $V$; viz. $V=\frac{1}{2} i \operatorname{Tr}(d P P d P)$ where $P(x)$ is the orthogonal projection onto $X_{x}$.

${ }^{13}$ I emphasize that (5) holds for the factor $\gamma(C)$ in (2) even if $E(s) \not \equiv 0$.

${ }^{14}$ Since $d V=0$ (i.e., $\operatorname{div} \tilde{V}=0$ if $V=\tilde{V}_{z} d x \wedge d y+\tilde{V}_{y} d z$ $\wedge d x+\tilde{V}_{x} d y \wedge d z$ ), away from $\overrightarrow{\mathrm{x}}=0$, (7) holds for any surface $S$ surrounding 0 .

${ }^{15}$ Since Berry is talking about integrating $(e, d e)$ along curves which he makes analogous to a vector potential, he talks about magnetic monopoles. Since we only care about (de, de) whose dual is divergenceless away from degeneracies, we do not use the magnetic monopole language. Since the dual may not have zero curl, electrostatic language is not appropriate. Since the sources have a sign, we still use the phrase "charge" for the coefficient of the delta function in $d[(d e, d e)]$ at singularities.
${ }^{16}$ One can show (see Avron, Seiler, and Simon, and Avron et al., Ref. 12) that such interpolations exist; indeed, that in the finite-dimensional case, the set of such interpolations is a dense open set in the set of all interpolations.

${ }^{17} \mathrm{~J}$. von Neumann and E. Wigner, Phys. Z. 30, 467 (1929); see also J. Avron and B. Simon, Ann. Phys. (N.Y.) 110, 85-110 (1978).

${ }^{18}$ These things will be discussed further in Avron, Seiler, and Simon, Ref. 12.

${ }^{19}$ Changing basis multiplies $C$ by a unitary and so $\sigma\left(x_{0}\right)$ is independent of basis. It does depend on an orientation of $R^{3}$ (order of $x, y, z$ ) but so do the TKN ${ }^{2}$ integers. Everything $\left(\sigma\right.$, the $\mathrm{TKN}^{2}$ integers, the sign of spanning surfaces for $C$ ) changes sign under change of orientation.

${ }^{20}$ The fact that the sum of the $\mathrm{TKN}^{2}$ integers is zero in the finite-dimensional case is made particularly transparent by these relations. The number of points of degeneracies of level $n$ and level $n+1$ is at least the absolute value of the sum of the first $n \mathrm{TKN}^{2}$ integers. 